\title{
Preparation and characterization of chitosan-g-poly (vinyl alcohol)/poly(vinyl alcohol) blends used for the evaluation of blood-contacting compatibility
}

\author{
Trong-Ming Don ${ }^{\mathrm{a}, *}$, Chia-Fong King ${ }^{\mathrm{b}}$, Wen-Yen Chiu ${ }^{\mathrm{b}}$, Ching-An Peng ${ }^{\mathrm{c}}$ \\ ${ }^{a}$ Department of Chemical and Materials Engineering, Tamkang University, Tamsui, Taipei County 25147, Taiwan, ROC \\ ${ }^{\mathrm{b}}$ Department of Chemical Engineering, National Taiwan University, Taipei 106, Taiwan, ROC \\ ${ }^{\mathrm{c} D e p a r t m e n t ~ o f ~ C h e m i c a l ~ E n g i n e e r i n g ~ a n d ~ M a t e r i a l s ~ S c i e n c e, ~ U n i v e r s i t y ~ o f ~ S o u t h e r n ~ C a l i f o r n i a, ~ C a l i f o r n i a, ~ C A ~ 90089, ~ U S A ~}$
}

Received 7 June 2005; revised 8 August 2005; accepted 18 August 2005

Available online 28 September 2005

\begin{abstract}
To increase the physicochemical compatibility between chitosan and poly(vinyl alcohol), chitosan- $g$-poly(vinyl alcohol)/poly(vinyl alcohol) (CS- $g$-PVA/PVA) blends were prepared via a two-step reaction. In the first step, chitosan- $g$-poly(vinyl acetate)/poly(vinyl acetate) (CS-g-PVAc/PVAc) blends were produced in situ by adding $100 \mathrm{~g}$ of VAc monomer to acetic aqueous solutions with different amounts of $\mathrm{CS}$, using a ceric ion as the initiator. The polymerization was carried out at $60{ }^{\circ} \mathrm{C}$ for $2 \mathrm{~h}$, where grafting of VAc monomers onto chitosan and homopolymerization of VAc monomers occurred simultaneously. Monomer conversion, grafting efficiency and grafting ratio were all measured by gravimetric analysis. In the second step, CS-g-PVA/PVA blends were obtained by converting PVAc chains to PVA through alcoholysis in an alkali methanol solution. After oven drying process, the morphology of CS- $g$-PVAc/PVAc and CS- $g$-PVA/PVA blends were observed to be particulate and dense membrane, respectively. The cellular and blood compatibility of pure PVA, pure chitosan, and CS$g$-PVA/PVA blends were tested separately by the viability of osteoblasts and the adhesion of platelets. Our results showed the cellular compatibility of PVA was improved due to the incorporation of chitosan. For platelet adhesion, pure PVA offered a good blood-contact property, while pure chitosan did very poor. However, interesting enough, the blends of a small amount of CS- $g$-PVA with PVA further improved the blood compatibility. An optimum composition of CS- $g$-PVA/PVA blend was determined in this study for its use in bloodcontacting biomedical devices.
\end{abstract}

(C) 2005 Elsevier Ltd. All rights reserved.

Keywords: Chitosan; Poly(vinyl acetate); Poly(vinyl alcohol); Osteoblast; Platelet adhesion

\section{Introduction}

Chitosan (CS) is a high-molecular-weight polysaccharide composed mainly of $\beta$ - $(1,4)$ linked 2-deoxy-2-amino-Dglucopyranose and partially of $\beta$ - $(1,4)$ linked 2-deoxy-2acetamido-D-glucopyranose. It is generally prepared by the partial deacetylation of chitin in a hot alkali solution. Because of several unique and interesting biological properties such as biocompatibility, biodegradability, and nontoxic properties, chitosan has been considered for the development

\footnotetext{
* Corresponding author. Tel.: +886 22629 3856; fax: +88622620 9887.

E-mail address: tmdon@mail.tku.edu.tw (T.-M. Don).
}

0144-8617/\$ - see front matter (C) 2005 Elsevier Ltd. All rights reserved. doi:10.1016/j.carbpol.2005.08.023 of membranes and fibers for hemodialysis, artificial skin and wound dressing, as well as scaffolds for tissue engineering and vehicles for drug and gene delivery (Amiji, 1995; Chandy \& Sharma, 1992; Francis \& Matthew, 2000; Ishihara et al., 2002; Kumar, Muzzarelli, Muzzarelli, Sashiwa, \& Domb, 2004). However, for applications that involve blood contact, chitosan promotes surface-induced thrombosis and embolization (Amiji, 1995; Chandy et al., 1992; Ishihara et al., 2002). When blood is in contact with thrombogenic materials, plasma proteins are first adsorbed on the surfaces, followed by the adhesion and activation of platelets. Upon activation, platelets secret their granular contents including adenosine diphosphate, thrombin and serotonin to activate other resting platelets as well as the coagulation reaction, therefore leading to thrombus formation (Anderson \& Kottke-Merchant, 1987). Several strategies reported to improve blood compatibility of chitosan are blending, 
grafting or crosslinking of synthetic polymers to chitosan (Amiji, 1995; Blair, Guthrie, Law, \& Turkington, 1987; Chandy et al., 1992; Chuang, Young, Yao, \& Chiu, 1999; Don, Hsu, \& Chiu, 2001; Don, King, \& Chiu, 2002a, b; Koyano, Koshizaki, Umehara, Nagura, \& Minoura, 2000; Minoura, Koyano, Koshizaki, Umehara, Nagura, \& Kobayashi, 1998; Nakatsuka \& Andrady, 1992).

One of the widely used polymers to be incorporated into chitosan is poly(vinyl alcohol), PVA, because of its biocompatible, nontoxic, and excellent mechanical properties. It exhibits minimal cell adhesion and protein adsorption (Burczak, Gamian, \& Kochman, 1996; Chuang et al., 1999; Ishihara et al., 2002; Minoura et al., 1998; Yang, Yao, Chang, \& Chen, 1996), and has been found to be biodegradable (Takasu, Aoki, Tsucyia, \& Okada, 1999). Simple blends of CS and PVA have good mechanical properties, and applications of these CS/PVA blends have been reported (Chandy et al., 1992; Koyano et al., 2000; Miya, Iwamoto, \& Mima, 1984; Nakatsuka et al., 1992; Wang, Turhan, \& Gunasekaram, 2004). Miya et al. (1984) suggested the enhancement of tensile strength of the blend may be due to the hydrogen bonding between hydroxyl groups of PVA and amine or hydroxyl groups of chitosan. However, CS and PVA have also been reported to be immiscible in several papers (Arvanitoyannis, Kolokuris, Nakayama, Yamamoto, \& Aiba, 1997; Chuang et al., 1999; Koyano et al., 2000; Lee, Kim, \& Kim, 1996; Minoura et al., 1998; Yang, Sua, Leub, \& Yang, 2004). Minoura et al. (1998) prepared hydrogels of CS/PVA blends and indicated that chitosan was enriched on the surface of gel membranes. This implied that a phase separation occurred in CS/PVA blends. Chuang et al. (1999) prepared a CS/PVA blend and showed that they were not miscible according to SEM and DSC analysis. Yang et al. (2004) prepared blends of CS and PVA in various ratios and treated with formaldehyde. They found that CS and PVA were not very compatible in the CS/PVA-blended hydrogel membranes. To enhance the CS/PVA compatibility and its ensuing properties for biomedical use, PVA was designed to be grafted onto chitosan in this study.

Graft copolymerization of chitosan with synthetic polymers endows desired properties to the hybrid materials. In addition, the formation of graft copolymer would enhance the compatibility of the two individual polymer components. Several methods can be utilized to initiate graft copolymerization, including electromagnetic radiation such as $\gamma$-ray and UV light, and free radical initiators such as azobisisobutyronitrile (AIBN) (Blair et al., 1987), iron (II)-hydrogen peroxide (Lagos and Reyes, 1998) and transition metal ions (Lopyrev, Voronkov, Baibordina, Shaglayeva, \& Rakhmatulina, 1979; Nagarajan, Sudhakar, \& Srinivasan, 1994; Qiu, Zhao, \& Dong, 1994). Among these, it has been shown that ceric ion is the most effective in grafting vinyl monomers onto a number of polysaccharides (Fernandez, Casinos, \& Guzman, 1990; Graczyk \& Hornof, 1988; Storey \& Goff, 1989). Only a few papers were found regarding the preparation of CS- $g$-PVA copolymer. Cho, Han, and Ko.
(2000) prepared a CS- $g$-PVA copolymer by grafting a chitosan oligomer (degree of polymerization of 9) onto PVA backbone chains by a coupling agent, $N$-methylolacrylamide. Kweon and Kang (1999) prepared CS-g-PVA copolymers by grafting water-soluble PVA onto chitosan directly using a ceric ion. The reaction mechanism was that the ceric ion preferentially split the vicinal diol (1,2-glycol) units present in few percents along the PVA chain, producing a limited number of chain segments carrying aldehyde groups at both ends or at one end. The terminal aldehyde groups were then reacted with the amino groups in chitosan, thus forming CS- $g$-PVA copolymers. However, A-B crosslinked copolymer was probably produced as well, as reported by Paradossi, Lisi, Paci, and Crescenzi (1996). They prepared a telechelic PVA obtained by periodate splitting of the 1,2-glycol unit in PVA (instead of using ceric ion) and subsequently used the telechelic PVA to crosslink chitosan molecules.

In this research, we adopted a different approach that a two-step reaction was employed to graft PVA chains onto a high-molecular-weight chitosan. In the first step, cerium ammonium nitrate (CAN) was used to initiate the graft copolymerization of vinyl acetate monomer (VAc) onto chitosan, forming a mixture of CS- $g$-PVAc copolymer and PVAc homopolymer. The reaction was carried out in a dispersion polymerization at $60^{\circ} \mathrm{C}$. The detailed reaction mechanism has been reported previously (Don et al., 2002a, b). In the second step, we further converted the synthesized CS- $g$-PVAc/PVAc to CS- $g$-PVA/PVA by alcoholysis of PVAc chains to PVA. Cellular and blood compatibility of various CS- $g$-PVA/PVA blends were examined to evaluate the potential for biomedical applications.

\section{Materials and methods}

\subsection{Materials}

Chitosan (Tokyo Chemicals, Inc., Tokyo) was purified before use. It was first dissolved into a $2 \%$ acetic acid solution and precipitated out by the addition of a concentrated $\mathrm{NaOH}$ solution. The precipitate was washed several times with a great amount of deionized water until the $\mathrm{pH}$ reached 7 . The degree of deacetylation of chitosan was found to be $86 \%$ by a colloid titration method (Toei \& Kohara, 1975). In this method, a specific amount of chitosan-acetic acid solution was titrated with $0.0025 \mathrm{~N}$ PVSK (potassium poly(vinyl sulfate), $\left(\mathrm{C}_{2} \mathrm{H}_{3} \mathrm{O}_{4} \mathrm{SK}\right)_{n}, n=1500$ or above), where $0.1 \%$ toluidine blue was used as an indicator. The viscosity average molecular weight $\left(\bar{M}_{v}\right)$ of chitosan, determined by a capillary viscometric method in a $0.2 \mathrm{M} \mathrm{AcOH} / 0.2 \mathrm{M}$ AcONa solution at $25^{\circ} \mathrm{C}$, was calculated to be $4.5 \times 10^{5}$ using the MarkHouwink equation $[\eta]=k \bar{M}_{v}^{\alpha}$, where $k$ and $\alpha$ were $1.38 \times$ $10^{-2}$ and 0.85 , respectively (Don et al., 2002a, b). VAc obtained from Acros Organics (Belgium) was distilled under reduced pressure. Only the distillate obtained at the middle 
stage of distillation was used for polymerization. CAN, $\left(\mathrm{Ce}\left(\mathrm{NH}_{4}\right)_{2}\left(\mathrm{NO}_{3}\right)_{6}\right)$, was a reagent-grade from Showa Chemical, Inc. (Tokyo, Japan). All the other chemicals were analytical-grade or above and used as received without further purification.

\subsection{Graft copolymerization}

The detail of the graft copolymerization was described elsewhere (Don et al., 2002a, b). Briefly, chitosan ( $\left.W_{\mathrm{CS}}\right)$ was dissolved in a $2 \%(\mathrm{w} / \mathrm{w})$ acetic acid solution and then $2.16 \times$ $10^{-2}$ mol of CAN initiator was added. Subsequently, $100 \mathrm{~g}$ of distilled VAc monomer $\left(W_{\mathrm{VAc}}\right)$ was added to the solution and the polymerization was carried out at $60^{\circ} \mathrm{C}$. After $2 \mathrm{~h}$, dispersion solution containing chitosan copolymer was obtained. To calculate monomer conversion $(X \%)$, the solution was dried to a constant weight $\left(W_{1}\right)$. To obtain grafting efficiency (GE\%) and grafting ratio (GR), PVAc homopolymer was removed by acetone extraction with a Soxhlet extractor (EYELA, Tokyo Rikakikai, Japan). After $72 \mathrm{~h}$ of extraction, the remaining chitosan- $g$-PVAc copolymer was dried and weighed $\left(W_{2}\right)$. Monomer conversion, grafting efficiency and grafting ratio were all calculated by the gravimetric method according to the equations listed below.

$$
\begin{aligned}
& X=\left(W_{1}-W_{\mathrm{CS}}\right) /\left(W_{\mathrm{VAc}}\right) \times 100 \\
& \mathrm{GE}=\left(W_{2}-W_{\mathrm{CS}}\right) /\left(W_{1}-W_{\mathrm{CS}}\right) \times 100 \\
& \mathrm{GR}=\left(W_{2}-W_{\mathrm{CS}}\right) /\left(W_{\mathrm{CS}}\right)
\end{aligned}
$$

\subsection{Alcoholysis of PVAc to PVA}

About $20 \mathrm{~g}$ of $\mathrm{NaOH}$ was dissolved in $20-\mathrm{mL}$ water, and subsequently $500-\mathrm{mL} \mathrm{CH}_{3} \mathrm{OH}$ was added into the solution. The alkali methanol solution was heated to $40{ }^{\circ} \mathrm{C}$ and stirred at $100 \mathrm{rpm}$. The previously prepared dispersion solution (CS$g$-PVAc/PVAc, $250 \mathrm{~mL}$ ) was added dropwise into the solution in $45 \mathrm{~min}$. The reaction was continued for another 15 min. The alcoholysis reaction proceeded immediately once the dispersion latex was added into the methanol solution and a sediment product was observed. After filtration, methanol was used to wash the reaction product. For the preparation of membrane, the reaction product was dissolved in $2 \mathrm{wt} \%$ of acetic acid solution and then dried in an oven at $50{ }^{\circ} \mathrm{C}$ for $48 \mathrm{~h}$ and then in a vacuum oven at $80{ }^{\circ} \mathrm{C}$ for another $12 \mathrm{~h}$.

\subsection{Structure analysis and evaluation of properties}

Structure analysis was carried out with a Fourier transform infrared (FTIR) spectrophotometer (JASCO300E, JASCO, Japan). To obtain FTIR spectra, sample was directly mounted on the sample holder and scanned from 4000 to $400 \mathrm{~cm}^{-1}$ for 32 times to average the signal with a resolution of $4 \mathrm{~cm}^{-1}$. A scanning electron microscope (S-800, Hitachi, Tokyo, Japan) at $20 \mathrm{kV}$ was used to observe the surface morphologies of sample membranes. All specimens were coated with a conductive layer of sputtered gold. A differential scanning calorimeter (TA 2010, TA Instrument, USA) was used to observe the thermograms of the sample membranes. Nitrogen gas was purged into the cell. Samples were placed in aluminum pans and scanned from -40 to $250{ }^{\circ} \mathrm{C}$ at a heating rate of $10^{\circ} \mathrm{C} / \mathrm{min}$. A mid-point method was employed to estimate the glass transition temperature $\left(T_{\mathrm{g}}\right)$.

\subsection{Cell culture and MTT assay}

Human osteoblast-like MG-63 cells derived from an osteosarcoma of a 14-year-old male (American Type Culture Collection, Rockville, MD, USA) were grown in Dulbecco's modified Eagle's medium (DMEM) supplemented with $10 \%$ fetal bovine serum (FBS), $2 \mathrm{mM} \mathrm{L-}$ glutamine, $10 \mathrm{mM}$ HEPES buffer, $100 \mathrm{U} / \mathrm{mL}$ Penicillin and $100 \mathrm{mg} / \mathrm{mL}$ Streptomycin. The prepared membranes with $15 \mathrm{~mm}$ in diameter were sterilized with UV radiation and placed in wells of a 24-well tissue culture polystyrene plate (Corning, Action, MA, USA). A sterilized silicone ring (15mm diameter) was placed on each of the tested samples in the wells to prevent them from floating. After UV radiation for $24 \mathrm{~h}$, the samples were sterilized in $70 \%$ alcohol overnight and rinsed three times with Dulbecco's phosphate buffer solution (DPBS). Aliquots $(1 \mathrm{~mL})$ of cell suspension with $1 \times 10^{5}$ cell $/ \mathrm{mL}$ were layered on the sample membranes. Cells were cultured in a humidified incubator balanced with $5 \% \quad \mathrm{CO}_{2}$ at $37{ }^{\circ} \mathrm{C}$. The viabilities of osteoblasts were determined by the MTT (3-[4,5dimethylthiazol-2-yl]-2,5-diphenyltetrazolium bromide; Thiazolyl blue) assay at $4 \mathrm{~h}, 1$ day, 2 days and 4 days of post-incubation. MTT reagent is a yellow substance which produces a dark blue formazan product when incubated with viable cells. Therefore, the level of the reduction of MTT into formazan can reflect the level of cell metabolism. At each period of cultivation, $100 \mu \mathrm{L}$ MTT (M-2128, Sigma, St Louis, MO, USA) solution was added to each well. After 3-h incubation at $37^{\circ} \mathrm{C}$, dimethyl sulfoxide (Nacalai Tesque, Kyoto, Japan) with the amount of $200 \mu \mathrm{L}$ was added to dissolve the formazan crystals. The dissolved solution was swirled homogeneously about $10 \mathrm{~min}$ by the shaker. The optical density of the formazan solution was detected by an ELISA plate reader (ELx 800, BIO-TEK, Winooski, VT, USA) at $570 \mathrm{~nm}$. All experiments were performed in triplicate and results were averaged.

\subsection{Platelet adhesion}

Platelet adhesion study was conducted to determine the blood compatibility of sample membranes. The procedure was similar to that used by Woodhouse and Brash (1992). A fixed amount of sample was first dissolved in an acidic 
solution. The $\mathrm{pH}$ of the solution was then adjusted to 6 or other values using $1 \mathrm{~N} \mathrm{HCl}$ and $1 \mathrm{~N} \mathrm{NaOH}$. The solution was poured into a test tube, and then dried in a rotary oven. A uniform film coated on inner wall of the tube was thus obtained. The polymer-coated tube was further dried in a vacuum oven at $80^{\circ} \mathrm{C}$ for $12 \mathrm{~h}$ to remove any possible residual solvent. Human whole blood was obtained from a healthy donor in a local hospital following regulatory protocols. Each $250 \mathrm{~mL}$ blood was added with $35 \mathrm{~mL}$ anticoagulant citrate phosphate dextrose adenine solution (Sigma, St Louis, MO, USA) and was stored at $1-6{ }^{\circ} \mathrm{C}$ without more than 1 week before test. About $1 \mathrm{~mL}$ of human blood was drawn into the polymer-coated test tube for platelet adhesion test. The tube was rotated at $120 \mathrm{rpm}$ in a shaking incubator at $37{ }^{\circ} \mathrm{C}$. After $1 \mathrm{~h}$ of incubation, the test tube was removed from the shaking incubator. The number of platelets left in the blood was counted immediately with an automated hematology analyzer (K-1000, Sysmex, Illinois, USA). Blood incubated in a glass tube without polymer deposit was used as a control. The final number of platelets adhering to the polymer film was expressed as a percent of the control, namely, relative index of platelet adhesion (RIPA) according to the following equation:

RIPA $=\frac{N_{\mathrm{m}}^{\prime \prime}}{N_{\mathrm{g}}^{\prime \prime}}=\frac{N_{\mathrm{w}}-N_{\mathrm{m}}}{N_{\mathrm{w}}-N_{\mathrm{g}}}$

where $N^{\prime \prime}{ }_{m}$ and $N^{\prime \prime}{ }_{g}$ are the number of adhered platelets on the sample membrane and control glass tube, respectively; $N_{\mathrm{w}}$ is the number of platelets in the blood before incubation; $N_{\mathrm{m}}$ and $N_{\mathrm{g}}$ are the number of remaining platelets in the blood after incubation in the polymer-coated and control glass tube, respectively. The lower the RIPA value is, the better blood compatibility is.

\section{Results and discussion}

\subsection{Preparation of CS- $g-P V A / P V A$ blends}

CS- $g$-PVA/PVA blends were prepared via a two-step reaction. For the graft polymerization of VAc monomer onto chitosan in the first step, the reaction system was observed to be a stable dispersion solution. Monomer conversion, grafting efficiency and grafting ratio listed in Table 1 are consistent with the results reported previously (Don et al., 2002a, b). After $2 \mathrm{~h}$ of polymerization, the monomer conversion was found to be $70-80 \%$, and slightly decreased with an increase of chitosan. This is because the viscosity of reaction solution increases with the increment of chitosan, thereby decreasing the diffusion rate of monomers. The grafting efficiency, representing the competition of graft copolymerization with homopolymerization for VAc monomer, increased with the amount of chitosan used. The reason is that there were more active sites available for the graft copolymerization as more chitosan was added. However, the
Table 1

Monomer conversion (X\%) grafting efficiency $(\mathrm{GE} \%)$ and grafting ratio (GR) of various reaction systems after $2 \mathrm{~h}$ at $60{ }^{\circ} \mathrm{C}$

\begin{tabular}{lllll}
\hline Sample & PVAc101 & PVAc105 & PVAc110 & PVAc115 \\
\hline Chitosan $(\mathrm{g})$ & 1 & 5 & 10 & 15 \\
VAc $(\mathrm{g})$ & 100 & 100 & 100 & 100 \\
X $(\%)$ & 81.5 & 77.9 & 74.8 & 71.2 \\
GE $(\%)$ & 4.13 & 16.9 & 28.5 & 34.2 \\
GR & 3.37 & 2.63 & 2.13 & 1.62 \\
\hline
\end{tabular}

grafting ratio decreased when a greater amount of chitosan was added. In the second step, the prepared CS- $g$-PVAc/ PVAc blends were further converted to CS- $g$-PVA/PVA blends via an alcoholysis reaction at $40{ }^{\circ} \mathrm{C}$. Fig. 1 shows the FTIR spectra of pure chitosan, CS- $g$-PVAc copolymer in PVAc101 (PVAc homopolymer being removed by acetone extraction) and CS- $g$-PVA/PVA blend (PVA101). Chitosan exhibits characteristic absorption peaks at $1648 \mathrm{~cm}^{-1}$ (amide I, $\mathrm{C}=\mathrm{O}$ stretching) and $1552 \mathrm{~cm}^{-1}$ (amide II, N-H bending) due to its amide groups. After polymerization and extraction of homopolymer from the reaction product, FTIR spectrum of the residual graft copolymer CS- $g$-PVA clearly reveals an additional absorption peak of ester group at $1733 \mathrm{~cm}^{-1}$ attributed to grafted PVAc chains. This demonstrates the copolymer structure of chitosan and PVAc. After alcoholysis of PVAc101 sample, the ester absorption peak disappeared completely, indicating the total conversion of PVAc chains to PVA chains. Furthermore, a shift of the amide I absorption peak in chitosan to a lower wavenumber was observed. It is speculated that there was some interaction between chitosan and PVA component. The same situations were observed for the other CS- $g$-PVA/PVA blends.

Based on the complete conversion of PVAc to PVA and the information of molecular weights of vinyl acetate

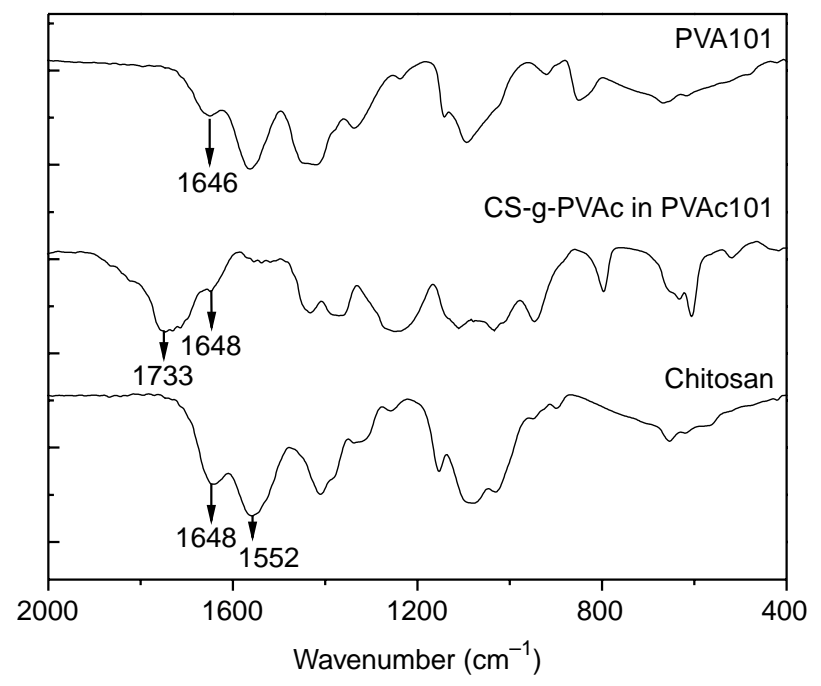

Fig. 1. FTIR spectra of pure chitosan, CS- $g$-PVAc copolymer in PVAc101 (PVAc homopolymer was removed by solvent extraction) and CS- $g$ PVA/PVA blend (PVA101). Grafting ratio in CS- $g$-PVAc and CS- $g$-PVA copolymers were 3.37 and 1.72 , respectively. 
Table 2

Weight of grafted PVA chains ( $g$-PVA) and PVA homopolymer (h-PVA) after alcoholysis reaction of CS- $g$-PVAc/PVAc blends

\begin{tabular}{lccll}
\hline Sample & PVA101 & PVA105 & PVA110 & PVA115 \\
\hline Chitosan (g) & 1 & 5 & 10 & 15 \\
g-PVAc (g) & 3.37 & 13.17 & 21.32 & 24.35 \\
h-PVAc (g) & 78.13 & 64.73 & 53.48 & 46.85 \\
g-PVA (g) & 1.72 & 6.74 & 10.91 & 12.46 \\
h-PVA (g) & 39.97 & 33.12 & 27.36 & 23.97 \\
GE & 16.9 & 28.5 & 34.2 \\
GR $_{\text {PVA }}$ & 4.13 & 1.35 & 1.09 & 0.83 \\
\hline
\end{tabular}

In addition, the percentage of grafted PVA chains to total PVA (GE $\left.\mathrm{GVA}_{\mathrm{P}}, \%\right)$ and the weight ratio of grafted PVA to chitosan $\left(\mathrm{GR}_{\mathrm{PVA}}\right)$ were also listed. A complete alcoholysis was confirmed by FTIR spectra. The weight of PVA chains thus was calculated by $W_{\mathrm{PVAc}} \times 44 / 86$, where 44 and 86 are the molecular weight of vinyl alcohol and vinyl acetate repeating unit, respectively. Grafting efficiency of PVA $\left(\mathrm{GE}_{\mathrm{PVA}}\right)$ equals to $W_{g \text {-PVA }} /\left(W_{g-}\right.$ $\left.\mathrm{PVA}+W_{\mathrm{h}-\mathrm{PVA}}\right) \times 100$ and grafting ratio of PVA $\left(\mathrm{GR}_{\mathrm{PVA}}\right)$ equals to $W_{g \text {-PVA }}$ $W_{\mathrm{CS}}$.

and vinyl alcohol repeating unit, the weights of PVA chains grafted onto chitosan ( $g$-PVA) and PVA homopolymer (hPVA) were calculated and listed in Table 2 . The total amount of PVA chains is not much different from one another, ranging from $41.69 \mathrm{~g}$ for the PVA101 blend to $36.43 \mathrm{~g}$ for the PVA115 blend. However, the total amount of chitosan increased from $1 \mathrm{~g}$ for the PVA101 to $15 \mathrm{~g}$ for the PVA115, and the grafting ratio of PVA chains decreased from 1.72 to 0.83 , respectively. It has been proved that when the PVAc chains are grafted onto chitosan chains in an acetic aqueous solution, a stable dispersion is obtained where hydrophobic PVAc chains aggregate inside the core and the hydrophilic chitosan chains migrate toward the outer shell of the particles (Don et al., 2002a, b). Therefore, the surface charge of dispersed particles is positive due to the cationic ammonium groups $\left(\mathrm{NH}_{3}^{+}\right)$in chitosan, since the amino groups are easily protonated in an acidic solution (i.e., $\mathrm{NH}_{2}+\mathrm{H}^{+} \rightarrow \mathrm{NH}_{3}^{+}$). The positive-charged chitosan on the surface thus provides the electrostatic and steric stabilization of particles. After oven drying, a particulate membrane is consequently obtained. This is because that the rigid chitosan chains on the particles' surface keep them from fusing together during the drying process. Unlike PVAc, PVA has a lot of hydroxyl groups and is a hydrophilic polymer. Therefore, converting PVAc to PVA would result in the change of affinity of the synthetic polymer to water. Both PVA and chitosan could extend their chains to a great extent in an aqueous solution. After drying, they could diffuse to each other, thus forming a dense film shown in Fig. 2. However, when the amount of added chitosan reached upper limits in the samples PVA110 and PVA115, voids were observed. This suggested that phase separation started to occur. The results in Table 2 give a plausible explanation, which shows that the grafting ratio decreases with an increasing amount of chitosan. The grafting ratio of PVA101 sample was about twice the value of PVA115 sample. The higher the grafting ratio, the more miscible the blend is. Therefore, the amount of grafted PVA
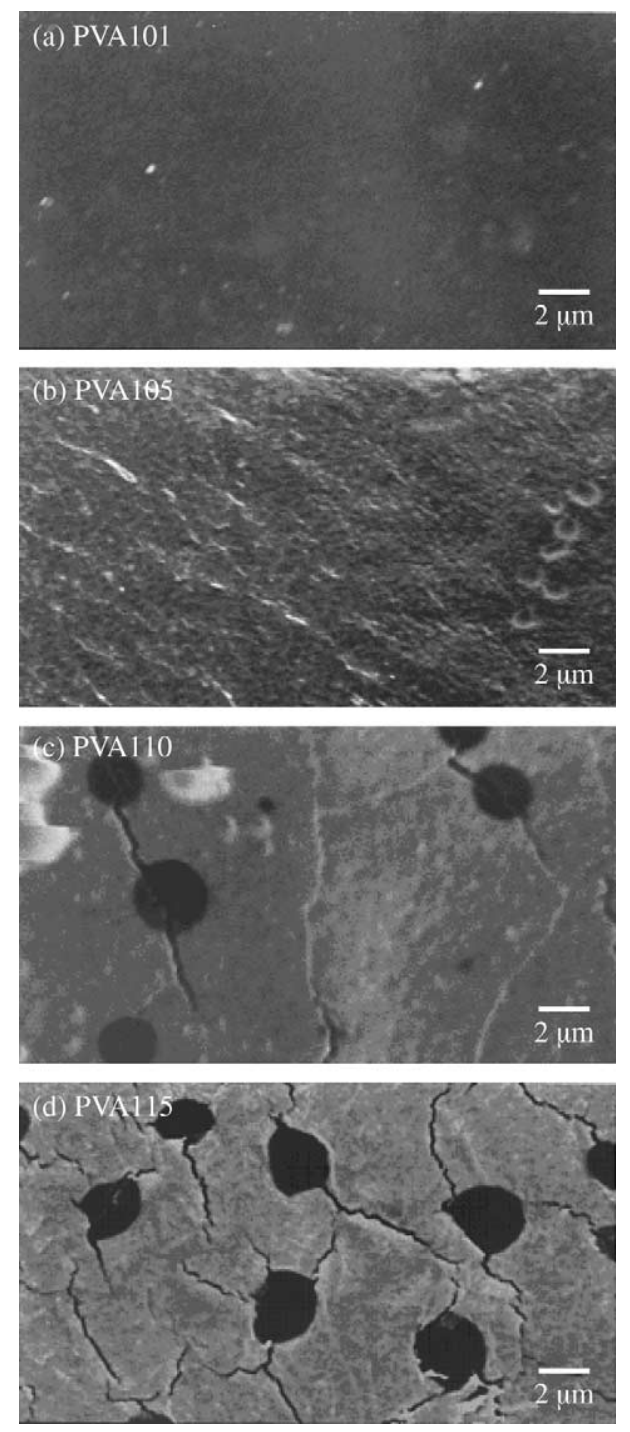

Fig. 2. SEM pictures of various CS-g-PVA/PVA samples with different amount of chitosan addition. (a) PVA101, (b) PVA105, (c) PVA110, and (d) PVA115. (PVA101 to PVA115 were obtained by alcoholysis of PVAc101 to PVAc115, respectively; and the number stands for 1 to $15 \mathrm{~g}$ of chitosan added to $100 \mathrm{~g}$ of VAc monomer for the reaction.)

chains in the samples PVA110 and PVA115 was not sufficient to render a complete miscibility between chitosan and PVA. Hence, chitosan started to separate out from the CS/PVA matrix. Judging from PVA115 blend having more voids than PVA110 blend, increasing chitosan amount and decreasing grafting ratio lead to phase separation to a large extent.

DSC was further used to investigate the miscibility of PVA and chitosan in order to support the appearance of SEM images. Figs. 3-5 show the changes of melting point $\left(T_{\mathrm{m}}\right)$, melting heat $\left(\Delta H_{\mathrm{m}}\right)$ and glass transition temperature $\left(T_{\mathrm{g}}\right)$ of PVA component in the blends, respectively. Pure PVA has $T_{\mathrm{m}}$ and $T_{\mathrm{g}}$ at 225 and $85^{\circ} \mathrm{C}$, respectively. However, no $T_{\mathrm{m}}$ and $T_{\mathrm{g}}$ were observed for chitosan in DSC due to its rigid structure and strong inter- and intra-hydrogen bonding. 


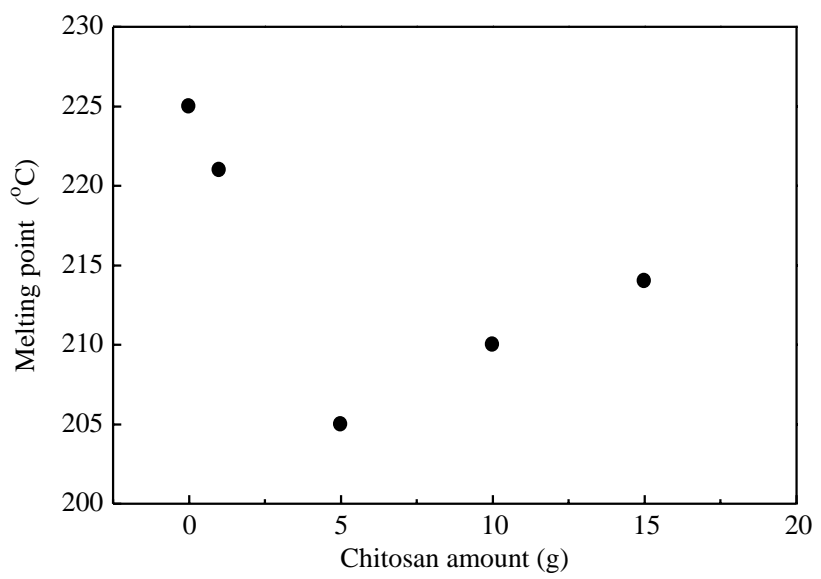

Fig. 3. Melting points of various CS-g-PVA/PVA samples with different amount of chitosan addition.

When chitosan was incorporated into the system to prepare CS- $g$-PVA/PVA blends, $T_{\mathrm{m}}, \Delta H_{\mathrm{m}}$ and $T_{\mathrm{g}}$ of PVA component all decreased gradually with the addition of chitosan up to $5 \mathrm{~g}$, illustrating these two polymers were miscible at this concentration range. The miscibility was due to the hydrogen bonding between PVA and chitosan, and enhanced by the grafting process. However, when the added amount of chitosan was increased to greater than $5 \mathrm{~g}$, transition temperature and melting heat started to increase, indicating phase separation began to develop. The DSC results are consistent with the SEM observations.

\subsection{Cell culture and MTT assay}

Fig. 6 shows the time course of formazan accumulation (\% of control) for pure chitosan, PVA and several copolymer membranes. Control group was the tissue culture treated polystyrene well with a silicone ring. Formazan absorbance indicates that osteoblasts in the culture well were able to convert the MTT into a blue formazan product. All the samples exhibited the same trends during 4-day

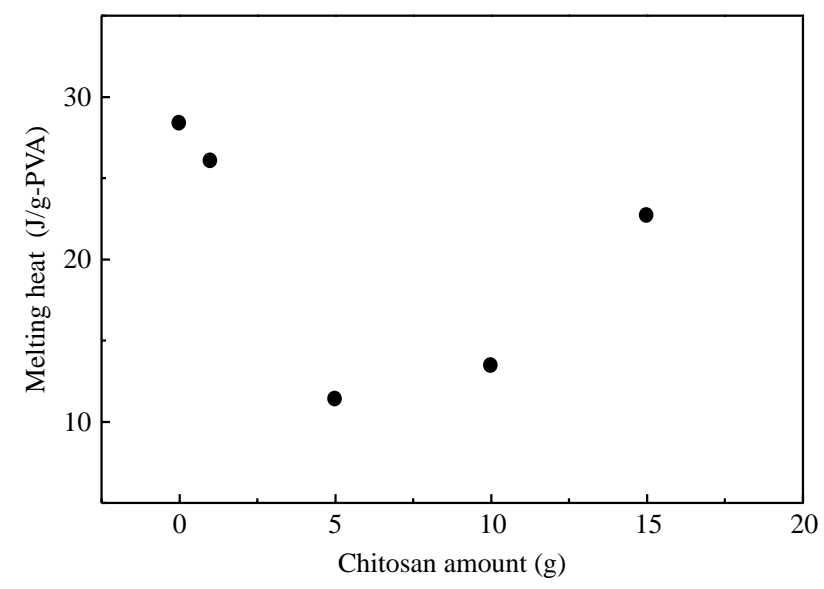

Fig. 4. Melting heat of various CS-g-PVA/PVA samples with different amount of chitosan addition.

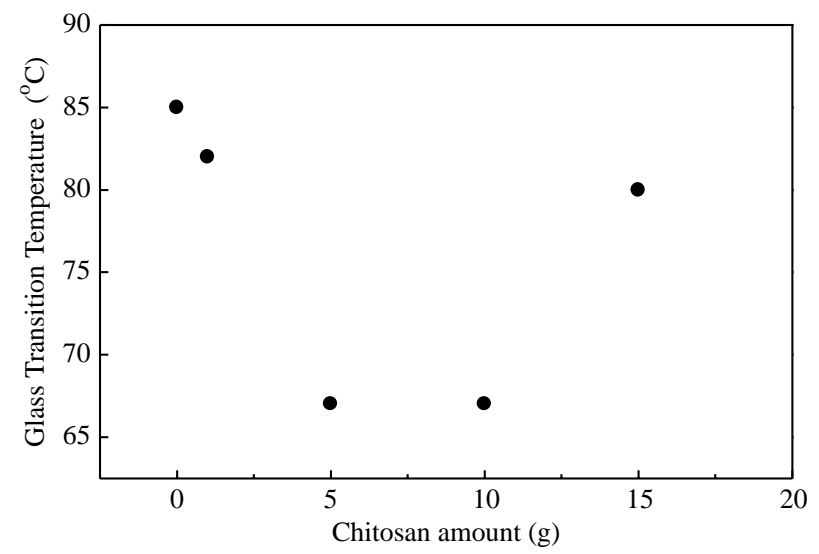

Fig. 5. Glass transition temperatures of various CS- $g$-PVA/PVA samples with different amount of chitosan addition.

cultivation. Cell growth reached its maximum at 1 day of culture and decreased afterwards. The results showed that good cellular compatibility was obtained for chitosan membrane. The absorbance ratios maintained at least $80 \%$ throughout the culture period. Due to its cationic nature, which is advantageous for cell adhesion, chitosan has been used as a biomaterial in tissue engineering and wound dressing. However, only 40-60\% of absorbance ratio was determined for pure PVA membrane. The cell viability on PVA membrane was not as good as that on chitosan probably because of its dense and smooth surface. However, when chitosan was introduced to modify PVA, the cell viability of osteoblasts was greatly improved. Even there was only one gram of chitosan addition (PVA101), the absorbance ratio could reach $87 \%$ after 1 day of culture. The incorporation of chitosan not only introduces cationic amino groups but also increases surface roughness and voids that facilitate cell adhesion and growth.

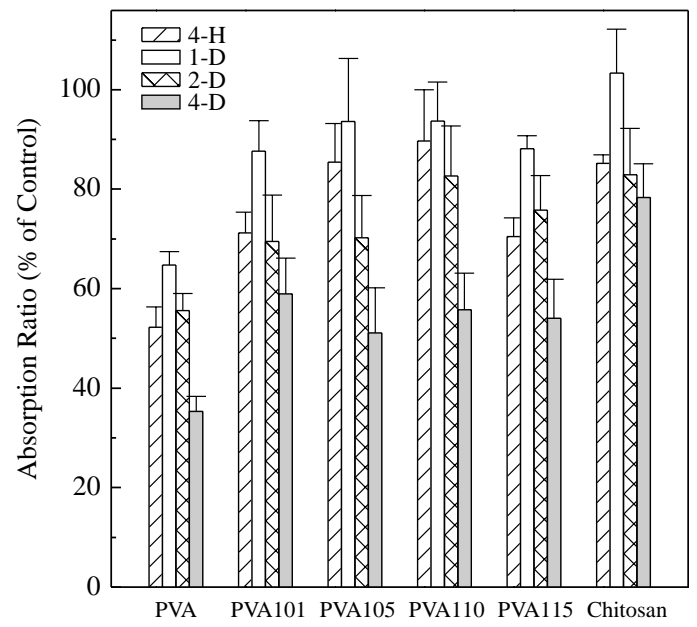

Fig. 6. Growth kinetics of osteoblasts on pure chitosan, poly(vinyl alcohol) (PVA) and various CS- $g$-PVA/PVA samples. Optical density at $570 \mathrm{~nm}$ $\left(\mathrm{OD}_{570}\right)$ was measured $(n=3)$ due to formazan absorbance (MTT assay): $4 \mathrm{~h}$ of incubation; 1 day of incubation; 2 days of incubation; 4 days of incubation. 


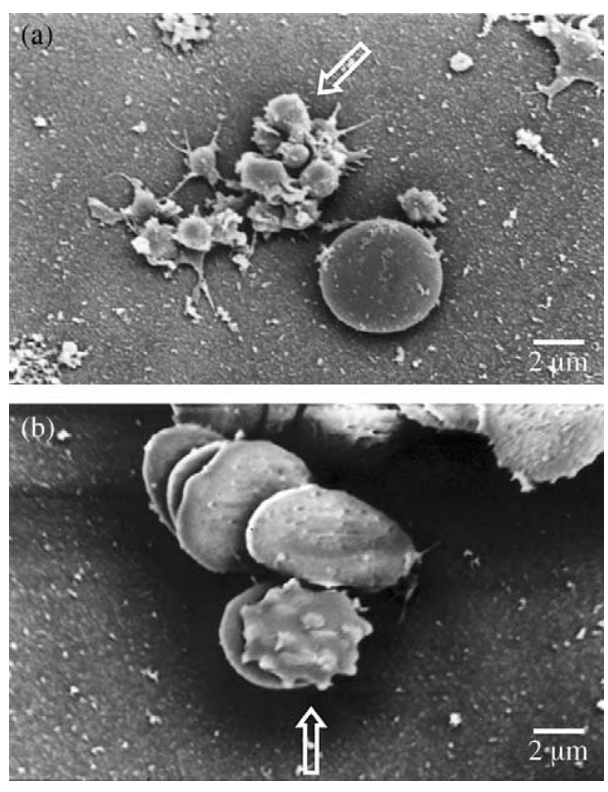

Fig. 7. Platelets were adsorbed on a CS- $g$-PVA/PVA membrane (PVA115), initially maintaining their discoid shape (2-4 $\mu \mathrm{m}$, indicated by white arrow) (a); platelets were fully activated, retracting their pseudopods to form circular or 'pancake' shape with the size about 4-6 $\mu \mathrm{m}$, indicated by white arrow (b).

\subsection{Platelet adhesion}

Though chitosan shows good cellular compatibility, it is highly thrombogenic also due to its cationic surface charge (Amiji, 1995; Chandy et al., 1992). The extent of platelet adhesion and surface-induced activation is considered as early indication of the thrombogenic potential of bloodcontacting biomaterials (Bair, DePalma, Goupil, \& Cohen, 1985). When in contact with membrane's surface (PVA115), platelets initially maintained their discoid shape $(2-4 \mu \mathrm{m})$, i.e. in the inactivated state (Fig. 7a). Upon activation, platelets extended their pseudopods and initiated the release of granular contents. When the platelets were fully activated, they retracted the pseudopods to form circular or 'pancake' shape with the size about 4-6 $\mu \mathrm{m}$ (Fig. 7b). The profiles of activated platelets were used to develop five activation stages as described by Lin, Ko, and Cooper (1994). In this study, relative index of platelet adhesion (RIPA) compared with the controlled glass tube was used to stand for blood compatibility. Platelet adhesion results for all CS- $g$-PVA/PVA blends are listed in Table 3. A high RIPA value was found for pure chitosan indicating poor blood compatibility; however, a good blood-contact property was observed for pure PVA. Surprisingly, the incorporation of a small amount of chitosan did not deteriorate the blood compatibility of PVA. On the contrary, it improved the blood-contact property. When $5 \mathrm{~g}$ of chitosan was added into the reaction system containing $100 \mathrm{~g}$ of VAc monomer to produce a CS- $g$-PVA/PVA blend (PVA105), a lowest RIPA value was achieved. After that, RIPA value increased with the addition of more chitosan, approaching to the value for pure chitosan. It is well known that many factors affect the formation of thrombosis including surface roughness, surface free energy, surface charge and its density, surface chemical structure, ratio of hydrophilic group to hydrophobic group, and the molecular orderness (crystallinity) on surface. Therefore, it is difficult to examine all these variables and their interactions that would affect the blood compatibility. However, the aforementioned structure analysis indicates that the platelet adhesion could be explained at least partly by the compatibility of CS and PVA component and the crystallinity of PVA. The DSC results showed that PVA105 membrane had the lowest crystallinity and melting point. The incorporation of a sufficient amount of chitosan disrupted the molecular packing of PVA chains. The decrease in the molecular orderness and crystallinity of PVA inhibited the platelet adhesion. However, as the added amount of chitosan increased, phase separation occurred and the crystallinity of PVA started to increase, in turn, increasing the platelet adhesion. It has to be pointed out other above-mentioned factors (e.g. surface roughness, surface chemical structure, etc.) could not be excluded, which are currently under investigation. It is also known that the platelet adhesion is related to the surface charge of membrane. Generally speaking, platelets easily deposit on a positive-charged surface than on a negative-charged surface. Kulik and Ikada (1996) found that the number of platelet adhesion on a positive-charged surface in P(AAmDMAEMA) was 4-fold of that on a neutral surface in PAAm; and was 6-fold of that on a negative-charged surface in $\mathrm{P}(\mathrm{AAm}-\mathrm{AAc})$. Therefore, solutions of CS-g-PVA/PVA blends having different $\mathrm{pH}$ values were prepared separately, since the extent of ionization and thus the number of positive-charged ammonium groups $\left(-\mathrm{NH}_{2} \rightarrow-\mathrm{NH}_{3}^{+}\right)$is affected by the $\mathrm{pH}$ value of solution. After coating on the surface of test tubes, they were tested for platelet adhesion and spreading. Table 4 demonstrated that as the number of positive-charged ammonium group increased by the lowering of $\mathrm{pH}$ value, RIPA value increased. The RIPA value for the sample prepared from the $\mathrm{pH} 3$ solution was 2.6 times of that for sample prepared from the solution with $\mathrm{pH}$ 9. In conclusion, PVA105 having a suitable cellular and blood compatibility offers the possibility of the usage for

Table 3

Relative index of platelet adhesion of various CS- $g$-PVA/PVA blends

\begin{tabular}{llllll}
\hline Sample & PVA & PVA101 & PVA105 & PVA110 & PVA115 \\
\hline RIPA & $0.418 \pm 0.021^{*}$ & $0.311 \pm 0.051$ & $0.139 \pm 0.050$ & $0.266 \pm 0.070$ & $0.533 \pm 0.067$ \\
\hline
\end{tabular}

*Standard deviation. 
Table 4

Relative index of platelet adhesion (RIPA) of a CS- $g$-PVA/PVA sample (PVA110) prepared in the solutions with different $\mathrm{pH}$ values

\begin{tabular}{lrrrr}
\hline $\mathrm{pH}$ & 3 & 5 & 7 & \multicolumn{1}{c}{9} \\
\hline RIPA & 0.644 & 0.434 & 0.400 & 0.256 \\
& $\pm 0.063 *$ & \pm 0.067 & \pm 0.072 & \pm 0.019 \\
\hline
\end{tabular}

*Standard deviation.

biomedical devices, which are directly in contact with blood.

\section{Conclusions}

In this study, CS- $g$-PVAc/PVAc blends were first prepared and then converted to CS-g-PVA/PVA blends via alcoholysis. After drying, both CS- $g$-PVAc/PVAc and CS- $g$-PVA/PVA membranes were obtained and characterized with two different morphologies-a particulate and a dense membrane, respectively. Phase separation started to develop as the amount of chitosan was increased up to $10 \mathrm{~g}$ and the grafting ratio decreased to ca. 1.09. The cellular and blood compatibility of CS- $g$-PVA/PVA blends were tested via osteoblast cell culture and platelet adhesion. The cellular compatibility of PVA was improved due to the incorporation of chitosan. Moreover, an optimum composition was attained for the CS- $g$-PVA/PVA blend to offer superior blood compatibility.

\section{Acknowledgements}

The authors wish to express their appreciation for the financial support of the National Science Council in Taiwan (Project No.: NSC 93-2313-B-032-002).

\section{References}

Amiji, M. M. (1995). Permeability and blood compatibility properties of chitosan-poly(ethylene oxide) blend membranes for hemodialysis. Biomaterials, 16, 593-599.

Anderson, J. M., \& Kottke-Merchant, K. (1987). Platelet interactions with biomaterials and artificial device. In D. F. Williams,Vol. 1 Blood compatibility (pp. 103-150). Boca Raton, FL: CRC Press Inc.

Arvanitoyannis, I., Kolokuris, I., Nakayama, A., Yamamoto, N., \& Aiba, S. (1997). Physico-chemical studies of chitosan-poly(vinyl alcohol) blends plasticized with sorbitol and sucrose. Carbohydrate Polymers, 34, 9-19.

Bair, R. E., DePalma, V. A., Goupil, D. W., \& Cohen, E. J. (1985). Human platelet spreading on substrata of known surface chemistry. Biomedical Material Research, 19, 1157-1167.

Blair, H. S., Guthrie, J., Law, T., \& Turkington, P. (1987). Chitosan and modified chitosan membranes I. Preparation and characterization. Journal of Applied Polymer Science, 33, 641-656.
Burczak, K., Gamian, E., \& Kochman, A. (1996). Long-term in vivo performance and biocompatibility of poly(vinyl alcohol) hydrogel microcapsules for hybrid-type artificial pancreas. Biomaterials, 17, 2351-2356.

Chandy, T., \& Sharma, C. P. (1992). Prostaglandin $E_{1}$-immobilized poly(vinyl alcohol)-blended chitosan membranes. Blood compatibility and permeability properties. Journal of Applied Polymer Science, 44(2), 2145-2156.

Cho, Y. W., Han, S. S., \& Ko, S. W. (2000). PVA containing chitooligosaccharide side chain. Polymer, 41, 2033-2039.

Chuang, W. Y., Young, T. H., Yao, C. H., \& Chiu, W. Y. (1999). Properties of the poly(vinyl alcohol)/chitosan blend and its effect on the culture of fibroblast in vitro. Biomaterials, 20, 1479-1487.

Don, T. M., Hsu, S. C., \& Chiu, W. Y. (2001). Structures and thermal properties of chitosan-modified poly(methyl methacrylate). Journal of Polymer Science, Polymer Chemistry Edition, 39(10), 1646-1655.

Don, T. M., King, C. F., \& Chiu, W. Y. (2002a). Preparation of chitosangraft-poly(vinyl acetate) copolymers and their adsorption of copper ion. Polymer Journal, 34, 418-425.

Don, T. M., King, C. F., \& Chiu, W. Y. (2002b). Synthesis and properties of chitosan-modified poly(vinyl acetate). Journal of Applied Polymer Science, 86, 3057-3063.

Fernandez, M. J., Casinos, I., \& Guzman, J. M. (1990). Effect of the way of addition of the reactants in the graft copolymerization of a vinyl acetate. Methyl acrylate mixture onto cellulose. Journal of Polymer Science, Polymer Chemistry Edition, 28, 2275-2292.

Francis, S. J. K., \& Matthew, H. W. T. (2000). Application of chitosanbased polysaccharide biomaterials in cartilage tissue engineering: A review. Biomaterials, 21, 2589-2598.

Graczyk, T., \& Hornof, V. (1988). Graft copolymerization of cellulose initiated by ceric salts: Effect of reaction conditions on the consumption of ceric ion. Journal of Polymer Science, Polymer Chemistry Edition, 26, 2019-2029.

Ishihara, M., Nakanishi, K., Ono, K., Sato, M., Kikuchi, M., Saito, Y., et al. (2002). Photocrosslinkable chitosan as a dressing for wound occlusion and accelerator in healing process. Biomaterials, 23, 833-840.

Koyano, T., Koshizaki, N., Umehara, H., Nagura, M., \& Minoura, N. (2000). Surface states of PVA/chitosan blended hydrogels. Polymer, 41, 4461-4465.

Kulik, E., \& Ikada, Y. J. (1996). In vitro platelet adhesion to nonionic and ionic hydrogels with different water contents. Biomedical Material Research, 30, 295-304.

Kumar, M.N.V. Ravi, Muzzarelli, R. A. A., Muzzarelli, C., Sashiwa, H., \& Domb, A. J. (2004). Chitosan chemistry and pharmaceutical perspective. Chemical Reviews, 104, 6017-6084.

Kweon, D. K., \& Kang, D. W. (1999). Drug-release behavior of chitosan-gpoly(vinyl alcohol) copolymer matrix. Journal of Applied Polymer Science, 74, 458-464.

Lagos, A., \& Reyes, J. (1998). Grafting onto chitosan. I. Graft copolymerization of methyl methacrylate onto chitosan with Fenton's reagent $\left(\mathrm{Fe}^{2+}-\mathrm{H}_{2} \mathrm{O}_{2}\right)$ as a redox initiator. Journal of Polymer Science, Polymer Chemistry Edition, 26, 985-991.

Lee, Y. M., Kim, S. H., \& Kim, S. J. (1996). Preparation and characteristics of $\beta$-chitin and poly(vinyl alcohol) blend. Polymer, 37, 5897-5905.

Lin, J. C., Ko, T. M., \& Cooper, S. L. (1994). Polyethylene surface sulfonation: Surface characterization and platelet adhesion studies. Journal of Colloid and Interface Science, 164, 99-106.

Lopyrev, V. A., Voronkov, M. G., Baibordina, E. N., Shaglayeva, N. S., \& Rakhmatulina, T. N. (1979). On the mechanism of initiation of polymerization by an oxidant-thiourea system. Journal of Polymer Science, Polymer Chemistry Edition, 17, 3411-3412.

Minoura, N., Koyano, T., Koshizaki, N., Umehara, H., Nagura, M., \& Kobayashi, K. (1998). Preparation, properties, and cell attachment/ growth behavior of $\mathrm{PVA} /$ chitosan-blended hydrogels. Materials Science and Engineering: C, 6, 275-280. 
Miya, M., Iwamoto, R., \& Mima, S. (1984). FT-IR study of intermolecular interactions in polymer blends. Journal of Polymer Science, Polymer Physics Edition, 22, 1149-1151.

Nagarajan, S., Sudhakar, S., \& Srinivasan, K. S. V. (1994). Block copolymerization initiated by $\mathrm{Mn}(\mathrm{III})$-poly(ethylene glycol) redox system—general features and kinetics. Colloid Polymer Science, 272, 777-783.

Nakatsuka, S., \& Andrady, A. L. J. (1992). Permeability of vitamin B-12 in chitosan membranes. Effect of crosslinking and blending with poly(vinyl alcohol) on permeability. Journal of Applied Polymer Science, 44, 17-28.

Paradossi, G., Lisi, R., Paci, M., \& Crescenzi, V. (1996). New chemical hydrogels based on poly(vinyl alcohol). Journal of Polymer Science, Polymer Chemistry Edition, 34, 3417-3425.

Qiu, K. Y., Zhao, J. B., \& Dong, J. H. (1994). Radical polymerization and graft polymerization with ceric ion and 3-phenylmethyl-2,4-pentanedione system. Polymer Bulletin, 32, 581-588.

Storey, R. F., \& Goff, L. J. (1989). Ceric ion initiation of vinylidene chloride from poly(vinyl alcohol). Macromolecules, 22, 1058-1064.
Takasu, A., Aoki, K., Tsucyia, M., \& Okada, M. J. (1999). New chitinbased polymer hybrids, 4: Soil burial degradation behavior of poly(vinyl alcohol)/chitin derivative miscible blends. Journal of Applied Polymer Science, 73, 1171-1179.

Toei, K., \& Kohara, T. (1975). A conductometric method for colloid titrations. Analytica Chimica Acta, 83, 59-65.

Wang, T., Turhan, M., \& Gunasekaram, S. (2004). Selected properties of pH-sensitive, biodegradable chitosan-poly(vinyl alcohol) hydrogel. Polymer International, 53, 911-918.

Woodhouse, K. A., \& Brash, J. L. (1992). Adsorption of plasminogen from plasma to lysine-derivatized polyurethane surfaces. Biomaterials, 13, 1103.

Yang, J. M., Sua, W. Y., Leub, T. L., \& Yang, M. C. (2004). Evaluation of chitosan/PVA blended hydrogel membranes. Journal of Membrane Science, 236, 39-51.

Yang, T. H., Yao, N. K., Chang, R. F., \& Chen, L. W. (1996). Evaluation of asymmetric poly(vinyl alcohol) membranes for use in artificial islets. Biomaterials, 17, 2139-2145. 